\title{
Platform-Intermediated Trade with Uncertain Quality
}

\author{
by \\ Benjamin E. Hermalin*
}

\begin{abstract}
Consider trade conducted via a platform, such as an online app store or a farmers market. The quality sellers choose for their products affects the surplus trade generates. Because the platform's profit depends on that surplus, the platform can have an incentive to regulate quality. This is true even if quality is observable at time of purchase or if sellers can develop reputations concerning quality. It is also true if the platform charges sellers only; that is, even if the platform has no direct interest in buyers' well being. (JEL: L14, L15, L22)

Keywords: Two-sided markets, platform-intermediated trade, private regulation of quality
\end{abstract}

\section{Introduction}

Apple and the Ithaca Farmers Market might seem related only insofar as the former is named for a fruit that one can buy at the latter. Yet both are trading platforms for sellers of Apple apps or, say, apple pies. An additional commonality is that they regulate product quality: both, for instance, have numerous quality requirements that sellers must meet and they will remove sellers who subsequently fail to meet these requirements.

Although the role of government as a regulator of quality (e.g., the Food and Drug Administration in the US) is long standing and well understood, the role of platforms as regulators has not, to the best of my knowledge, been explored. This paper is an initial foray into the topic. Like many such forays, it raises more questions than it answers and principally demonstrates more research is warranted.

Unlike the government, a platform's income depends on the regulations it sets. To wit, because its income derives from capturing either some of the consumer surplus or profits (revenues) or both that trade generates, its objectives vis-à-vis regulation derive from that income. Moreover, the platform's pricing of access to buyers, sellers, or both will in turn affect the incentives sellers have to provide quality.

To get a sense of the complexity of the issues, consider what might seem obvious truths when the platform regulates quality:

1. If the platform's revenues derive from capturing a fraction of buyers' consumer surplus, then the platform will wish to set a higher quality standard than if its revenues derive from capturing a share of sellers' profits.

${ }^{*}$ University of California, Berkeley. The author grateful acknowledges the financial support of the Thomas and Alison Schneider Distinguished Professorship in Finance. 
2. If buyers observe quality at time of purchase, then the platform will have little incentive to regulate quality, especially if it earns its revenues by charging sellers.

3. Ceteris paribus, a platform does strictly better when buyers know product quality at time of purchase than when they do not.

4. A situation of observable quality is equivalent to a setting in which quality is unobservable to buyers, but there is repeated play and all sellers are highly patient.

As will be seen, however, these "truths" are at worst false and at best incomplete.

In what follows, the platform is assumed to use one of three forms of pricing: a flat access charge to buyers, a flat access charge to sellers, or a so-called agency arrangement in which sellers pay a fraction of their revenue to the platform. Although some platforms arguably use a combination of these (e.g., Apple could be seen as charging buyers an access fee via the price they pay for their iPhones or iPads and it charges app developers a commission of 30\%), most platforms use only one and the analysis, correspondingly considers just that. ${ }^{1}$ In particular, platforms tend to charge sellers and not buyers. For this reason, much of what follows focuses on the platform's pricing to sellers.

The model is set forth in the next section. Section 3 then considers the benchmark case of observable quality, where it is shown that agency distorts quality downward, but all sellers will be active; whereas charging flat access fees results in higher quality being provided by active sellers, but can lead to the exclusion of some sellers. Even when flat access fees are charged, the sellers' market power means quality can fail to be welfare maximizing due to the familiar problem that such a seller sets quality on the basis of the benefit it provides the marginal rather than average buyer (see, e.g., Spence, 1975).

Section 4 assumes quality is unobservable, but that the platform can set a minimum quality level. If the platform charges sellers a flat access fee, then the platform's profit is the same in this regime as it was when quality is observable. This is because the platform sets the quality level to maximize the profit of the marginal seller (i.e., the one on the cusp between being active and shutting down). The platform's profit with a flat access fee also exactly equals the profit the platform would now earn under agency given unobservable quality. It turns out that this could be a higher profit than the platform would earn under agency with observable quality but no minimum level. An implication is that a platform employing agency earns a greater profit setting a minimum quality standard even with observable quality than if it didn't set such a standard.

In many models of quality provision, repeated play can overcome informational asymmetries given sufficiently patient parties (see, e.g., Klein and Leffler, 1981). Hence, one might guess that in a repeated version of the model, quality observability is irrelevant given sufficient patience. This is false here; see Section 5. To wit, when the platform charges sellers for access, it undermines their incentives to maintain a reputation for high quality. So even with the most patient sellers, quality under repeated play when the platform charges a flat access fee will never be as high as it would be given observable

\footnotetext{
${ }^{1}$ As discussed in depth infra, an obvious topic for future research is when the platform can use multiple and more sophisticated forms of pricing.
} 
quality (at least for a subset of sellers). Even if the platform imposes minimum quality standards, the quality decisions of sellers will vary from what they would be were quality observable: some will deliver higher quality, others lower quality.

As noted, the paper only scratches the surface in understanding the role of a platform as private regulator. Throughout the paper, various potential extensions are noted, as well as limitations to the current approach. In addition, Section 6 offers additional thoughts about the platform as regulator.

\section{Model}

A continuum of buyers wish to acquire products offered by a continuum of sellers. Without loss, let the measure of each side be one. Each buyer wishes to purchase at most one unit from each seller irrespective of his dealings with other sellers; that is, the sellers are not competing. To trade, buyers and sellers must use a monopoly platform.

Let $s \in[0,1]$ index the sellers. Denote the proportion of sellers with an index (type) not exceeding $s$ by $\Psi(s), \Psi:[0,1] \rightarrow[0,1]$. Assume $\Psi$ is continuously differentiable and let $\psi$ denote its derivative (the density function). Assume $\psi(s)>0$ for all $s \in(0,1)$.

Seller $s$ incurs a cost of $c(q, s)$ to develop a product of quality $q$. This is a fixed cost of production or, in the repeated version of the model, a fixed per-period cost of maintaining quality. Consistent with many of the motivating examples, assume marginal production costs are zero. ${ }^{2}$ Assume quality is chosen from $[0, \infty) .{ }^{3}$ Assume the following about $c$ : (i) it is twice continuously differentiable in its arguments; (ii) $c(0, s)=0$ for all $s$; (iii) $c(\cdot, s)$ is increasing and strictly convex for all $s$; (iv) $0=\partial c(0, s) / \partial q \equiv c_{q}(0, s)$ for all $s$ and $\lim _{q \rightarrow \infty} c_{q}(q, s)=\infty$ for all $s$; and

$$
c_{q s}(q, s) \equiv \frac{\partial^{2} c(q, s)}{\partial q \partial s}>0
$$

An implication of assumption (ii) is

$$
c_{s}(0, s) \equiv \frac{\partial c(0, s)}{\partial s}=0 .
$$

Assumption (iv) rules out corner solutions at zero or infinite quality. Note the use of subscripts to denote partial derivatives.

A consequence of these assumptions is that

$$
c_{s}(q, s)=c_{s}(0, s)+\int_{0}^{q} c_{q s}(z, s) d z=\int_{0}^{q} c_{q s}(z, s) d z>0
$$

\footnotetext{
${ }^{2}$ This seems a reasonable accurate assumption for digital goods. That said, a zero marginal cost is not simply a normalization: were it more than negligible, then, under agency, the commission ("tax") rate the platform sets would directly affect a seller's profit-maximizing sales. That effect is absent (de minimis) when marginal cost is zero (negligible).

${ }^{3}$ As will become evident, one can impose an upper bound on quality. The assumption is that if there is an upper bound it never binds.
} 
where the first equality follows from the fundamental theorem of calculus, the second from (1), and the inequality from assumption (v). Expression (2) states that, holding quality constant, seller cost increases with seller index (type).

For each seller $s$ there is an associated index set $\mathcal{N}_{s}=[0,1]$ and each buyer has a unique index (type), $n_{s}$, within that set. As will seen, a buyer's index number relates to his preferences for that seller's product. If buyers don't pay for access, it is irrelevant how a given buyer's index numbers vary across sellers. When buyers purchase access, it is convenient to assume they are homogenous with respect to the decision to acquire access. To achieve this, while still allowing them heterogenous preferences with respect to any given seller, assume:

Assumption 1 A buyer's index (type) within $\mathcal{N}_{s}$ is drawn independently of his index (type) in $\mathcal{N}_{s^{\prime}}, s \neq s^{\prime}$. All draws are from the uniform distribution. Buyers learn their types (preferences) for the various sellers' products only after acquiring access (although they know the distributions ex ante), but before buying products.

The timing in Assumption 1 could be reasonable insofar as a consumer could have a vague sense of how much he would like various different apps for a smartphone, say, and it is only after purchasing the phone and investigating the apps that his true preferences reveal themselves. ${ }^{4}$ Assuming types are drawn from the uniform is without loss because one can make a change of variables with respect to the mapping from types to preferences.

Denote by $\beta\left(n_{s}, q_{s}\right)$ buyer $n_{s}$ 's gross benefit from a unit of a good of quality $q_{s}$. Note $\beta$ is not seller dependent - this simplifies the analysis and facilitates a focus on the role of quality. Buyer $n_{s}$ 's interaction with seller $s$ adds $\beta\left(n_{s}, q_{s}\right)-p_{s}$ to his total utility if he buys at price $p_{s}$ and zero otherwise. Observe sellers are limited to linear tariffs. When unneeded, the subscript $s$ will be omitted to avoid notational clutter.

Take $\beta$ to be non-negative and twice continuously differentiable in its arguments. Assume that $\beta(\cdot, q)$ is non-increasing for any given $q$. Buyers like higher quality: $\beta(n, q)>$ $\beta\left(n, q^{\prime}\right)$ if $q>q^{\prime}$ for all $n \in[0,1)$. These assumptions imply that $\beta(0, q)>0$ if $q>0$. To avoid corner solutions, assume $\beta_{q}(n, 0)>0$ for all $n \in[0,1)$. Assume a diminishing marginal benefit of quality $\left(i . e ., \beta_{q q}(n, q) \leq 0\right)$. Going forward assume either that

$$
\beta_{n q}(n, q)>-\frac{\beta_{q}(n, q)}{n} \text { for all } n>0 \text { and } q
$$

(as but one example, (3) holds if $\beta$ is additively separable in $n$ and $q$ ) or that

$$
\beta(n, q)=(\theta q+\kappa) b(n),
$$

$b$ twice differentiable and non-increasing, with $b(0)>0$, and $\theta>0$ and $\kappa \geq 0$ constants. Given known or anticipated quality $q$, buyer $n$, facing price $p$, buys if and only if

$$
\beta(n, q)-p \geq 0 .
$$

\footnotetext{
${ }^{4}$ Admittedly, this is a less reasonable assumption concerning people's preferences for apples and tomatoes, but then farmers markets tend not to charge buyers an access fee.
} 
Because $\beta(\cdot, q)$ is non-increasing, if (5) holds for $n$ it holds for $n^{\prime}<n$. Because a purchaser acquires one unit only and type is distributed uniformly, $\beta(\cdot, q)$ is an inverse demand curve conditional on quality. So that sellers have unique profit-maximizing quantities to sell, assume $n \mapsto n \beta(n, q)$ is a strictly log concave mapping for all $q$.

Assume the timing of the game is as follows: 1) the platform announces its policies and prices; 2) sellers choose their qualities, decide whether to acquire access to the platform, and post their prices; 3) buyers decide to acquire access; 4) buyers learn their types with respect to each product; and 5) buyers make their purchase decisions.

Assume that the platform cannot discriminate among users on a given side of the market. In fact, in what follows, the platform will be restricted to fairly simple tariffs. More sophisticated pricing is a topic left for future work.

\section{Benchmark: Observable Quality}

As a benchmark, suppose initially that buyers know (observe) the quality of each seller's wares before purchase.

Having chosen quality $q$, a seller can sell $n$ units at price $\beta(n, q)$. As discussed in detail below, her access to the platform could be contingent on her paying the equivalent of an ad valorem tax. Let $\tau$ denote the tax rate. Her profit is, thus,

$$
(1-\tau) \beta(n, q) n-c(q, s)
$$

Previously made assumptions ensure there exists at least one pair of profit-maximizing sales, $n^{*}$, and quality, $q^{*}$. To speed the analysis, assume the pair is unique. To that end, note the problem of maximizing profit can be solved in two steps. In the first, determine the profit-maximizing sales for an arbitrary quality. Because $n \beta(n, q)$ is strictly log concave, a unique solution exists, $n^{*}(q) .{ }^{5}$ A key feature of $n^{*}(\cdot)$ is:

LEMma 1 Given condition (3), $n^{*}(\cdot)$ is an increasing function (when not equal to one). Given condition $(4), n^{*}(\cdot)$ is a constant with respect to quality. ${ }^{6,7}$

Turning to the second step: solve

$$
\max _{q}(1-\tau) \beta\left(n^{*}(q), q\right) n^{*}(q)-c(q, s) .
$$

Using the envelope theorem, it is readily seen that the second derivative of that objective function is

\footnotetext{
${ }^{5}$ Because $q$ is endogenous, $n^{*}$ ultimately varies with seller type, $s$, and tax rate, $\tau$. When this dependency needs highlighting, I will sometimes write $n^{*}(s, \tau)$ rather than $n^{*}\left(q^{*}(s, \tau)\right)$; such shorthand should not, I hope, cause confusion.

${ }^{6}$ Proofs not given in the text can be found in the appendix.

${ }^{7}$ As the proof makes clear, were (3) reversed, then $n^{*}(\cdot)$ would be decreasing - higher quality would correspond to fewer sales. For the sake of brevity, this case is not explored here.
} 
(8) $(1-\tau)\left(\beta_{n q}\left(n^{*}(q), q\right) n^{*}(q)+\beta_{q}\left(n^{*}(q), q\right)\right) n^{* \prime}(q)$

$$
+(1-\tau) \beta_{q q}\left(n^{*}(q), q\right) n^{*}(q)-c_{q q}(q, s)
$$

The top line of (8) is non-negative given Lemma 1 and conditions (3) and (4). The bottom line is negative given the assumptions about $\beta_{q q}$ and $c_{q q}$. The assumption that $q^{*}$ be unique can, thus, be seen as an assumption that the top line of (8) is never too big (note it is zero if $\beta(n, q)=(\theta q+\kappa) b(n))$.

As a benchmark result:

Proposition 1 Assume observable quality. Assume either (3) or (4). Then the seller's profit-maximizing sales are non-increasing in the commission (tax) rate it faces and with respect to its index. Profit-maximizing quality is decreasing in the commission rate and with respect to index. If (3) holds and profit-maximizing sales are an interior solution, sales decrease with the commission rate and with respect to index.

The usual Spence (1975) quality distortion exists. To wit, even absent the distortions that a positive tax rate induces, a seller's quality can differ from the level that would maximize welfare holding sales equal to $n^{*}$. A seller chooses $q$ to maximize (7), whereas maximizing welfare given $n^{*}$ sales would have her maximize

$$
\int_{0}^{n^{*}} \beta(n, q) d n-c(s, q)
$$

The first-order condition is

$$
\int_{0}^{n^{*}} \beta_{q}(n, q) d n-c_{q}(s, q)=0
$$

By the intermediate value theorem, there is some $\hat{n} \in\left(0, n^{*}\right)$ such that the lefthand side of (9) equals $n^{*} \beta_{q}(\hat{n}, q)-c_{q}(q, s)$. A seller's profit-maximizing quality level solves the first-order condition $n^{*} \beta_{q}\left(n^{*}, q\right)-c_{q}(q, s)=0$ (having set $\tau=0$ ). If marginal benefit of quality increases with type (i.e., $\left.\beta_{n q}>0\right)$, then $\beta_{q}\left(n^{*}, q\right)>\beta_{q}(\hat{n}, q)$; so comparing first-order conditions and recalling that cost is convex, it follows that $q^{*}$ exceeds the $q$ that solves (9). Conversely, if marginal benefit of quality falls with type (i.e., $\beta_{n q}<0$ ), then $\beta_{q}\left(n^{*}, q\right)<\beta_{q}(\hat{n}, q) ; q^{*}$ is less than the $q$ that solves (9). If $\beta_{n q}=0$, as would be true if $\beta(n, q)=q+b(n)$, then $q^{*}$ equals the $q$ that solves (9). To summarize:

Proposition 2 Holding sales fixed at the profit-maximizing level, a seller's provision of quality relative to the corresponding welfare-maximizing level is greater if higher index buyers have a greater marginal benefit of quality than lower index buyers (i.e., if $\beta_{n q}>0$ ); less if higher index individuals have a lower marginal benefit of quality than lower index buyers (i.e., if $\beta_{n q}<0$ ); and equal if higher index individuals have the same marginal benefit of quality as lower index buyers (i.e., if $\beta_{n q}=0$ ). 
Let $\pi(s, \tau)$ denote a seller's equilibrium profits; that is, the value of (6) at the profitmaximizing level of sales and quality. The envelope theorem then entails

$$
\pi_{s}(s, \tau)=-c_{s}\left(q^{*}, s\right)<0,
$$

where the inequality follows from (2). When $\tau=0$, it helps reduce notational clutter to write $\pi(s)$ rather than $\pi(s, 0) .^{8}$

\subsection{Access to the Platform and Welfare}

Now, consider access. Suppose initially that buyers have free access and the platform charges only sellers for access. There are two prominent models of pricing access: a flat access fee or "agency" (i.e., the platform takes a percentage of sellers' revenues). ${ }^{9}$

Consider a flat access fee $\alpha$. If seller $s$ is willing to pay it (i.e., $\pi(s) \geq \alpha$ ), then (10) implies any seller with a lower index would as well. Consequently, the platform's pricing problem is isomorphic to choosing the $s$ that maximizes

$$
\Psi(s) \pi(s)
$$

( $\Psi$, recall, is the distribution of seller types). Previously made assumptions ensure the platform's objective has at least one maximizer. Furthermore, although nothing ensures a unique maximum, the Maximum Theorem (see, e.g., Sundaram 1996, p. 235) guarantees that there is a largest maximizer of (11); call it $s^{*}$. In what follows, assume that the platform sets its access price equal to $\pi\left(s^{*}\right) \equiv \alpha^{*}$; that is, conditional on maximizing its profit, the platform prices to induce the greatest number of sellers to join it. Note, among all such equilibria, that one yields the greatest welfare.

The alternative pricing regime is agency: the platform collects a proportion of the seller's price or, equivalently, her revenue. Let $\tau$ denote the commission ("tax") rate. The platform sets $\tau$ to maximize

$$
\left.\tau \int_{0}^{1} \beta\left(\left(n^{*}(z, \tau)\right), q^{*}(z, \tau)\right)\right) n^{*}(z, \tau) \psi(z) d z,
$$

where $n^{*}(s, \tau)$ and $q^{*}(s, \tau)$ are a type- $s$ seller's profit-maximizing level of sales and quality, respectively, given $\tau$.

\footnotetext{
${ }^{8}$ This will be a general convention. Hence, e.g., the shortly to be introduced $q^{*}(s, \tau)$ will be written as $q^{*}(s)$ when $\tau=0$.

${ }^{9}$ As will become evident, the platform would almost always do better if it could set both an access fee and a "tax" rate (i.e., use a two-part tariff). Because platform pricing is not the focus of this paper, the use of two-part tariffs is left for future extensions. In terms of real life, while Apple charges a modest fee for the technology needed to develop apps for its devices (\$99/year as of this writing), its principal source of revenue is from the $30 \%$ commission (tax rate) it charges; that is, Apple uses essentially a pure agency model. At the other extreme, the Ithaca Farmers Market simply charge sellers a fixed access fee.
} 
Without further assumptions, it is ambiguous as to whether the platform's profit is greater charging sellers a flat access fee or under agency. ${ }^{10}$

What is unambiguous, however, is that if seller $s$ would operate under either regime and marginal benefit of quality doesn't increase with buyer type (i.e., $\beta_{n q} \leq 0$ ), then the welfare generated by seller $s$ is greater if the platform charges a flat access fee than if it employs agency: welfare generated by this seller is

$$
W(s, \tau) \equiv \int_{0}^{n^{*}(s, \tau)} \beta\left(n, q^{*}(s, \tau)\right) d n-c\left(q^{*}(s, \tau), s\right) .
$$

It follows that

$$
\begin{aligned}
\frac{\partial W(s, \tau)}{\partial \tau}=\beta\left(n^{*}(s, \tau), q^{*}(s, \tau)\right) \overbrace{\frac{\partial n^{*}(s, \tau)}{\partial \tau}}^{\leq 0} & \\
& +\underbrace{\left(\int_{0}^{n^{*}(s, \tau)} \beta_{q}\left(n, q^{*}(s, \tau)\right) d n-c_{q}\left(q^{*}(s, \tau), s\right)\right)}_{\geq 0} \underbrace{\frac{\partial q^{*}(s, \tau)}{\partial \tau}}_{<0} \leq 0
\end{aligned}
$$

where the signs of $\partial n^{*} / \partial \tau$ and $\partial q^{*} / \partial \tau$ follow from Proposition 1 and the sign of the expression in large parentheses follows because $q^{*}(s, \tau)$ does not exceed the welfaremaximizing quality level by Proposition 2. It cannot be that both terms in (12) labeled with a weak inequality are zero - the expression in the large parentheses can be zero only if $\beta(n, q)$ is additively separable in $n$ and $q$ (i.e., of the form $b(n)+\gamma(q))$, but then

$$
\frac{\partial^{2}}{\partial n \partial q} \beta(n, q) n=\gamma^{\prime}(q) n>0,
$$

which implies $\partial n^{*}(s, \tau) / \partial \tau>0$ (see proof of Proposition 1 for details). To summarize:

Proposition 3 Assume seller quality is observable. Conditional on its operating regardless of the platform's choice of profit-maximizing access regime, a seller generates greater welfare under a flat-fee regime than under agency if marginal benefit of quality doesn't increase with type (i.e., $\beta_{n q} \leq 0$ ).

What Proposition 3 does not establish is that total welfare is greater with a flat access fee than under agency. The reason is that more sellers could be active under agency than under a flat-fee regime. Indeed, all sellers will be active in agency - even if the platform set $\tau=1$, no seller would strictly wish to exit (although all would choose zero quality) $;^{11}$ whereas, by picking a suitable distribution function, it is straightforward

\footnotetext{
${ }^{10}$ Suppose that $\Psi$ is the uniform distribution, let $c(q, s)=(s+1) q^{2}$, and let $\beta(n, q)=$ $(q+\kappa)(1-n)$. If $\kappa=1 / 2$, then a flat fee outperforms agency. If $\kappa=1$, the reverse is true. Calculations available from author upon request.

${ }^{11} \mathrm{~A}$ corner solution in which the platform sets $\tau=1$ is not realistic and, thus, of no interest. Assume, going forward, that such a solution is never profit maximizing for the platform.
} 
to generate examples in which $s^{*}<1$. On the other hand, for certain distributions and parameter configurations, it can be shown that the platform would, under a flat-fee regime, set $s^{*}=1$, so all sellers are active. ${ }^{12}$ In such cases, the following corollary holds:

COROllary 1 Assume seller quality is observable. If, under the platform's profitmaximizing flat-fee regime, all sellers are active (i.e., $\left.s^{*}=1\right)$, then total welfare is greater under that regime than it would be under the platform's profit-maximizing agency regime if marginal benefit of quality doesn't increase with type (i.e., $\beta_{n q} \leq 0$ ).

Even if fewer sellers are active under the flat-fee regime, their average quality will be higher:

Corollary 2 Assume seller quality is observable. Comparing equilibria under a flatfee regime and agency, average quality of sellers active in the former will be greater than in the latter.

An alternative is that the platform charges the buyers a flat access fee, $A$. Given the assumption that the buyers are ex ante identical at the time they make their access decision, the platform would optimally set $A$ equal to their individual expected consumer surpluses. The surplus buyer $n_{s}$ derives from seller $s$ is

$$
\mathrm{CS}_{s}\left(n_{s}\right)=\left\{\begin{array}{l}
\beta\left(n_{s}, q^{*}(s, \tau)\right)-\underbrace{\beta\left(n^{*}(s, \tau), q^{*}(s, \tau)\right)}_{p_{s}}, \text { if } n_{s} \leq n^{*}(s, \tau) \\
0, \text { if } n_{s}>n^{*}(s, \tau)
\end{array} .\right.
$$

It follows that

$$
A=\int_{0}^{\hat{s}} \int_{0}^{n^{*}(s, \tau)} \mathrm{CS}_{s}(n) d n \psi(s) d s
$$

where $\hat{s}$ is the index of the marginal seller given the platform's pricing to sellers.

Determining the platform's prices is more involved if it can charge both sides. In particular, it will charge lower fees to sellers (and thus accommodate more sellers) when it can charge buyers than when it cannot (assuming $s^{*}<1$ ). This reflects the familiar access externality of two-sided markets. ${ }^{13}$ At this juncture, these tradeoffs are not especially germane and an analysis, thus, omitted for the sake of brevity.

\section{Unobservable Quality with Minimum Quality Standards}

Suppose, now, buyers cannot observe the quality of a seller's wares before purchase.

Assume, for the moment, that the platform is unable to enforce any quality level. Let $q^{a}$ denote the quality that buyers anticipate a seller will provide. The seller can,

\footnotetext{
${ }^{12}$ This would, for instance, be true if $\beta(n, q)=(1-n)+q, c(q, s)=(s+1) q^{2} / 2$, and $s$ is distributed uniformly on $[0,1]$.

${ }^{13}$ See, e.g., Rochet and Tirole (2006), who refer to this as a "membership" externality.
} 
thus, sell $n$ units at price $\beta\left(n, q^{a}\right)$, yielding a profit of

$$
(1-\tau) \beta\left(n, q^{a}\right) n-c(q, s) .
$$

Clearly, the seller's best response with respect to quality is $q=0$. Of course, in equilibrium, this must be anticipated by buyers; hence, $q^{a}=0$ in equilibrium.

Suppose that the platform can impose a quality level. Specifically, assume that the platform can pick a common quality level, $\hat{q}$, and ensure that all sellers on the platform offer at least that quality. It is readily seen that no seller would provide quality greater than $\hat{q}$ : for any anticipated $q^{a}$ by buyers, its objective, should it operate at all, is to maximize (14) subject to the constraint $q \geq \hat{q}$. The solution is $q=\hat{q}$; this is the quality offered by sellers who operate. In equilibrium, therefore, $q^{a}=\hat{q}$; that is, buyers anticipate the quality level that the platform mandates.

Given a fixed quality level, each active seller chooses her sales to maximize $\beta(n, \hat{q}) n$ (this is true regardless of $\tau$ ). The solution is $n^{*}(\hat{q})$. Observe, inter alia, this establishes:

LEMMA 2 When quality is unobservable, but the platform can set a minimum quality level, then all active sellers have the same number of sales and earn the same revenue.

A consequence of this lemma is that, because seller cost is increasing with index holding quality fixed (recall expression (2)), if a type- $s$ seller finds it profitable to operate given mandated quality $\hat{q}$ and tax rate $\tau$, then so too must all sellers with a lower index.

Suppose the platform employs a flat-fee regime. The access price equals the profit of the marginal seller, $s$, given quality $\hat{q}$. As just noted, all sellers with an index $s^{\prime}<s$ also acquire access. So if $\hat{\pi}$ is the profit of the marginal seller, $s$, the platform's profit is $\hat{\pi} \Psi(s)$. Holding $s$ fixed, it follows that the platform does best to mandate quality to maximize $\hat{\pi}$; that is, it will set $\hat{q}=q^{*}(s)$. In determining the marginal seller, the platform's problem is again choosing $s$ to maximize (11) where $\pi(s)$ is again

$$
\pi(s)=\beta\left(n^{*}(s), q^{*}(s)\right) n^{*}(s)-c\left(q^{*}(s), s\right) .
$$

Because the platform's optimization program is the same, the solution will be the same: the marginal seller will again be $s^{*}$. The difference, though, is all sellers now produce quality $q^{*}\left(s^{*}\right)$. To summarize:

Proposition 4 Assume quality is unobservable to buyers, but the platform can mandate a minimum level. In the equilibrium of a flat-fee regime, the platform's profit and the set of sellers who are active are the same as when quality was observable, but active sellers only produce quality equal to the profit-maximizing quality of the marginal seller (i.e., seller $\left.s^{*}\right)$. All active sellers but $s^{*}$ sell less than they would were quality observable.

The last sentence of Proposition 4 follows from Lemma 1

Proposition 4 is reminiscent of Spence (1975): a monopolist chooses quality to maximize the benefit of its marginal customer. Here, though, the customer is a seller and the quality is what if offers, rather than receives. 
Consider agency. The platform's program is

$$
\begin{gathered}
\max _{\tau, \hat{q}, \hat{s}} \tau \Psi(\hat{s}) \beta\left(n^{*}(\hat{q}), \hat{q}\right) n^{*}(\hat{q}) \\
\text { subject to }(1-\hat{s})\left((1-\tau) \beta\left(n^{*}(\hat{q}), \hat{q}\right) n^{*}(\hat{q})-c(\hat{q}, \hat{s})\right)=0,
\end{gathered}
$$

where (16) is the constraint that the marginal seller, $\hat{s}$, just breakeven. Because (15) increases in $\tau$, the platform does best to set

$$
(1-\tau) \beta\left(n^{*}(\hat{q}), \hat{q}\right) n^{*}(\hat{q})-c(\hat{q}, \hat{s})=0 .
$$

Rearranging that equality yields:

$$
\tau \beta\left(n^{*}(\hat{q}), \hat{q}\right) n^{*}(\hat{q})=\beta\left(n^{*}(\hat{q}), \hat{q}\right) n^{*}(\hat{q})-c(\hat{q}, \hat{s}) ;
$$

that is, the revenue the platform earns from each seller equals the profit of the marginal seller were she not subject to a tax. That last quantity is the same $\hat{\pi}$ considered above, which, plugging into (15), means the platform's objective is to maximize $\hat{\pi} \Psi(s)$. This is the same program it faced with a flat access fee. The following is immediate:

Proposition 5 Assume quality is unobservable to buyers, but the platform can mandate a minimum quality. In the equilibrium of an agency regime, the platform's profit and the set of sellers who are active are the same as they would be under the profit-maximizing flat-fee regime when quality is observable.

Because under agency with observable quality all sellers are active, one implication of Proposition 5 is to show that a consequence of unobservable quality is that fewer sellers are active. This might, at first glance, appear to be a "lemons effect" (Akerlof, 1970), but it is in fact quite different: it is the high-quality sellers who remain active and the exiting of the low-quality sellers is due to their effective exclusion by the platform.

Another implication of Proposition 5 stems from the earlier made point that it is ambiguous whether the platform earns greater profit using agency or a flat fee when quality is observable. It is, therefore, possible that a platform (exogenously) limited to agency earns greater profit when quality is unobservable than when it is observable:

COROLlary 3 Assume quality is unobservable to buyers, but the platform can mandate a minimum quality. Suppose the platform is limited to using agency. Then it is possible that the platform's profit is greater than if quality were observable to buyers (and the platform doesn't or is unable to set a minimum quality level).

The parenthetical remark in Corollary 3 raises the question: would a platform limited to agency, but able to set a minimum quality level, wish to do so even if quality were observable? The answer is yes:

Proposition 6 Assume quality is observable to buyers and the platform is limited to using agency. Then the platform profits by imposing a binding minimum quality level. 
Proof: Let $\tau$ be the profit-maximizing commission rate assuming no minimum standard. Because $c_{q}(0, s)=0$ for all $s, \beta_{q}>0$, and $\tau<1$ (see footnote 11 supra),

$$
(1-\tau) \beta\left(n^{*}\left(q^{*}(1, \tau)\right), q^{*}(1, \tau)\right) n^{*}\left(q^{*}(1, \tau)\right)-c\left(q^{*}(1, \tau), 1\right)>0 .
$$

Hold $\tau$ fixed, but consider a minimum standard $\hat{q}>q^{*}(1, \tau)$. Because sellers determine $n$ and $\beta_{q}>0$, it follows, using revealed preference, that

$$
\begin{aligned}
(1-\tau) \beta\left(n^{*}(\hat{q}), \hat{q}\right) n^{*}(\hat{q}) \geq(1-\tau) & \beta\left(n^{*}\left(q^{*}(1, \tau)\right), \hat{q}\right) n^{*}\left(q^{*}(1, \tau)\right) \\
& >(1-\tau) \beta\left(n^{*}\left(q^{*}(1, \tau)\right), q^{*}(1, \tau)\right) n^{*}\left(q^{*}(1, \tau)\right) .
\end{aligned}
$$

It follows from (17) and (18) that there exist $\hat{q}>q^{*}(1, \tau)$ such that

$$
(1-\tau) \beta\left(n^{*}(\hat{q}), \hat{q}\right) n^{*}(\hat{q})-c(\hat{q}, 1) \geq 0 .
$$

In other words, imposing such a minimum standard while maintaining the commission rate would not cause any sellers to exit. ${ }^{14}$ Given continuity, the minimum standard must bind on an interval of sellers $[\bar{s}, 1]$, which has positive measure because $\bar{s}<1$. The minimum standard raises the platform's profit:

$$
\begin{aligned}
\tau \int_{0}^{\bar{s}} \beta\left(n^{*}\left(q^{*}(s, \tau)\right), q^{*}(s, \tau)\right) n^{*}\left(q^{*}(s, \tau)\right) \psi(s) d s+\tau \int_{\bar{s}}^{1} \beta\left(n^{*}(\hat{q}), \hat{q}\right) n^{*}(\hat{q}) \psi(s) d s \\
>\tau \int_{0}^{1} \beta\left(n^{*}\left(q^{*}(s, \tau)\right), q^{*}(s, \tau)\right) n^{*}\left(q^{*}(s, \tau)\right) \psi(s) d s
\end{aligned}
$$

where the inequality follows by the logic behind (18).

What if the platform derives revenue solely from access fees charged buyers? As a first step, it is worth starting with the case $\beta(n, q)=b(n)+q$. Expression (13) becomes

$$
\mathrm{CS}_{s}(n)=\left\{\begin{array}{l}
b(n)-b\left(n^{*}(q)\right), \text { if } n \leq n^{*}(q) \\
0, \text { if } n>n^{*}(q)
\end{array}\right.
$$

Observe consumer surplus is directly independent of quality - sellers are able to capture via their pricing all benefit quality produces. Quality matters to consumer surplus only so far as it affects sales; in particular, if $b^{\prime}(n)<0$ and $n^{*}(q)<1$, quality increases sales, which means an indirect increase in consumer surplus. If $b^{\prime}(n)=0$ or $n^{*}(q)=1$ for relevant values of quality, then there would be no effect and the access fee, $A$, would

\footnotetext{
${ }^{14}$ Note the importance of the assumptions that $c_{q}(0, s)=c(0, s)=0$ to this conclusion: if there were a corner solution to the worst $(s=1)$ type seller's quality decision or if there were a fixed cost of production independent of quality, then (17) need not be a strict inequality, so imposing a binding minimum quality constraint could induce exit of some sellers.
} 
therefore also be independent of quality. Hence, as this example illustrates, the platform could be indifferent to the sellers' quality and would, therefore, have no incentive to impose minimum quality. As such, this example illustrates that, while a monopoly platform wants to maximize the net benefits the side it charges derives from trade with the other side, this does not translate into the platform necessarily caring about the quality that side receives. What matters is buyers' net, not gross benefit.

Indeed, this logic leads to the following. Define

$$
\mathrm{CS}(n, q \mid m)=\beta(n, q)-\beta(m, q) .
$$

Limiting attention to values for which consumer surplus is positive, an improvement in quality from $q^{\prime}$ to $q$, holding $n$ constant, changes consumer surplus by

$$
\begin{gathered}
\operatorname{CS}\left(n, q \mid n^{*}(q)\right)-\operatorname{CS}\left(n, q^{\prime} \mid n^{*}\left(q^{\prime}\right)\right) \\
=\left(\operatorname{CS}\left(n, q \mid n^{*}(q)\right)-\operatorname{CS}\left(n, q^{\prime} \mid n^{*}(q)\right)\right)+\left(\operatorname{CS}\left(n, q^{\prime} \mid n^{*}(q)\right)-\operatorname{CS}\left(n, q^{\prime} \mid n^{*}\left(q^{\prime}\right)\right)\right) \\
=\int_{q^{\prime}}^{q}\left(\beta_{q}(n, z)-\beta_{q}\left(n^{*}(q), z\right)\right) d z-\int_{n^{*}\left(q^{\prime}\right)}^{n^{*}(q)} \beta_{n}\left(x, q^{\prime}\right) d x \\
=-\int_{q^{\prime}}^{q} \int_{n}^{n^{*}(q)} \beta_{n q}(x, z) d x d z-\int_{n^{*}\left(q^{\prime}\right)}^{n^{*}(q)} \beta_{n}\left(x, q^{\prime}\right) d x
\end{gathered}
$$

(note the use of the fundamental theorem of calculus). The second term in the last line of (19) is non-negative because $\beta_{n} \leq 0$ and $n^{*}(\cdot)$ is non-decreasing. Because $n^{*}(q)>n$ and $q>q^{\prime}$, the sign of the first term on that line is the same as $-\beta_{n q}$. Consequently, a sufficient condition for consumer surplus to increase with quality is that marginal benefit of quality fall with buyer type. If marginal benefit of quality rises with buyer type, then the effect of increased quality on consumer surplus is ambiguous.

As this discussion makes clear, no general predictions can be made about how the platform would set the minimum quality level when it charges buyers versus when it charges sellers. It is, however, sufficient to establish:

Proposition 7 Assume quality is unobservable to buyers, but the platform can mandate a minimum quality. Circumstances exist-among them when buyer benefit is additively separable in type and quality and all buyers purchase in equilibrium-such that the platform has an incentive to set a higher quality standard when it charges only sellers for access than when it charges only buyers for access.

\section{Quality Sustained Through Reputation}

It is long been known that sellers of experience goods may be able to develop reputations for providing high-quality goods when sellers play repeatedly (see, e.g., Klein and Leffler, 1981). Suppose, now, that sellers play repeatedly. Specifically, assume that each seller is infinitely lived and either plays with the same set of buyers each period or each generation 
of buyers knows the history of past play. Each period repeats the stage game analyzed above; in particular, each seller is free to adjust her quality each period if she wishes.

To be precise, assume the sequence of play for each period's stage game is as above, except each active seller announces her quality (her announcement need not be truthful) when she posts her price; and, at the end of the stage, the quality each active seller actually provided becomes commonly known (think, e.g., of that period's buyers posting comments or ratings). Let $\delta \in(0,1)$ denote the sellers' discount factor.

In what follows, the focus will be on stationary equilibria (i.e., all parties make the same plays each period). Further, attention will be limited to grim-trigger strategies: should a seller's actual quality fall short of her claimed quality, then all buyers will forever after anticipate that she will play her best response for a one-period game (i.e., the stage game were it played once). As noted in the previous section, her best response, given buyers cannot observe quality prior to purchase, is to provide the minimum permissible quality: zero if the platform sets no minimum and the minimum otherwise. Conversely, buyers believe that if a seller has always provided quality at least as good as her claimed quality, then she will continue to do so going forward. Assume that buyers honestly report the quality they experience (i.e., their online reviews are truthful).

As the earlier analysis makes clear, even the simple model considered so far can be difficult to analyze. To make the analysis more tractable, assume:

Assumption $2 \beta(n, q)=(1+q / B) b(n)$, where $B=\max _{n} b(n) n=b\left(n^{*}\right) n^{*}$ and $b(\cdot)$ is such that $\beta$ satisfies all previous assumptions; in particular, $b(0)>0 .{ }^{15}$

As a consequence, if buyers anticipate a seller will deliver quality $q^{a}$, then it is profitmaximizing for that seller to set its price to be $\left(1+q^{a} / B\right) b\left(n^{*}\right)$, which will induce all buyers with index less than $n^{*}$ to buy; hence, the seller's revenue will be $q^{a}+B$.

\subsection{Platform Imposes No Quality Control}

Assume sellers pay a flat access fee of $\alpha$ each period, but no minimum quality is set.

Suppose that a seller has maintained a reputation for delivering her promised quality. Her revenue will be $B+q$, where $q$ is promised quality. Further, if it is profit maximizing to actually provide promised quality $q$ in a given period, it is profit maximizing to do so every period; hence, her discounted payoff from playing that strategy is

$$
\frac{1}{1-\delta}(B+q-c(q, s)-\alpha)
$$

Suppose, instead, that a seller has ruined her reputation, so buyers expect her to offer quality 0 if she is active. Given such expectations, her best response is to supply quality 0 if active. Given Assumption 2, she will be active if and only if $B \geq \alpha$. Define $\pi_{0} \equiv \max \{0, B-\alpha\}$. Her discounted payoff after ruining her reputation is $\pi_{0} /(1-\delta)$.

\footnotetext{
${ }^{15}$ The analysis that follows does not require $B=b\left(n^{*}\right) n^{*}$, only that $\beta$ be of the form given in expression (4); it is simply notationally convenient to have $1 / \theta=b\left(n^{*}\right) n^{*}$ and $\kappa=1$.
} 
An alternative to delivering promised quality is to deviate to a lower quality. Given that ruins her reputation, she may as well deviate to zero quality. Because buyers don't observe her deviation, they will still pay $(1+q / B) b\left(n^{*}\right)$ that period. The seller will sell to $n^{*}$ buyers and earn revenue $B+q$.

Putting all this together, a seller's quality promise is credible if and only if

$$
\frac{1}{1-\delta}(B+q-c(q, s)-\alpha) \geq B+q-\alpha+\frac{\delta}{1-\delta} \pi_{0}
$$

LEMMA 3 In equilibrium, a seller's per-period profit after ruining her reputation is zero (i.e., $\pi_{0}=0$ ); equivalently, $\alpha \geq B$ in equilibrium.

Proof: Suppose not. Because a seller can shutdown, $\pi_{0} \geq 0$. Hence, the supposition is $\pi_{0}>0$ or, equivalently, $\alpha<B$ (recall $B$ is positive given $b(0)>0$ ). It follows, therefore, that the platform could raise $\alpha$ without affecting the set of sellers who are active, which would increase its profit. Consequently, there can be no equilibrium in which $\alpha<B$.

In light of the lemma, (20) can be rewritten as

$$
q-c(q, s) \geq(1-\delta) q+\delta(\alpha-B)
$$

It is useful to define

$$
\underline{q}(s)=\underset{q}{\operatorname{argmax}} \delta q-c(q, s) .
$$

The above assumptions ensure a solution to that maximization program exists and is unique. Furthermore, $\underline{q}(s)>0$ because $\delta>0$ and $c_{q}(0, s)=0 ; \underline{q}(s)<q^{*}(s)$ because $\delta<1 ; \underline{q}(\cdot)$ is decreasing because $c_{q s}>0$; and, because $0 \neq \operatorname{argmax}_{q} \delta q-c(q, s)$,

$$
\delta \underline{q}(s)-c(\underline{q}(s), s)>0
$$

for all $s$. Finally, invoking the envelope theorem, it is readily seen that the lefthand side of (22) is a decreasing function of $s$ given that $c_{s}>0$.

Lemma 4 Fix an access fee, $\alpha$. If

$$
\delta \underline{q}(s)-c(\underline{q}(s), s)<\delta(\alpha-B),
$$

then a type-s seller is inactive. Otherwise, she is active and produces quality

$$
q^{e}(s) \equiv \max \left\{q \in\left[\underline{q}(s), q^{*}(s)\right] \mid \delta q-c(q, s) \geq \delta(\alpha-B)\right\} .
$$

LEMma 5 There exists an $\widetilde{s} \in(0,1]$ such that only sellers with an index in $[0, \widetilde{s}]$ are active, each active seller s provides quality $q^{e}(s)$, and the access fee is

$$
\alpha=\underline{q}(\widetilde{s})+B-\frac{c(\underline{q}(\widetilde{s}), \widetilde{s})}{\delta} .
$$


These lemmas lead to the main result of this section:

Proposition 8 Consider a situation of infinitely repeated play. Suppose that the platform charges sellers a fixed access fee. In equilibrium a positive measure, if not all, active sellers deliver less quality than they would were quality observable.

Proof: Consider seller $\widetilde{s}$ defined by (25). By construction, the only quality level for $\widetilde{s}$ to promise that is credible (satisfies expression $(21))$ is $\underline{q}(\widetilde{s})$. As shown earlier, $\underline{q}(\widetilde{s})<q^{*}(\widetilde{s})$. By continuity, there is an interval of seller types for whom $q^{e}<q^{*}$.

Given the assumed benefit function, $\beta_{n q}(n, q)=b^{\prime}(n) / B \leq 0$. Proposition 2(ii) or (iii) applies, which means even were quality observable, the sellers (weakly) provide less than the welfare-maximizing level. It follows from Proposition 8 that

COROLlary 4 Consider a situation of infinitely repeated play. Suppose that the platform charges sellers a fixed access fee. In equilibrium a positive measure, if not all, active sellers deliver less quality than the welfare-maximizing amount.

Because $q^{*}(1)-c\left(q^{*}(1), 1\right)>0$, there must exist a $\bar{\delta}<1$ such that

$$
q^{*}(1)-c\left(q^{*}(1), 1\right) \geq(1-\delta) q^{*}(1)
$$

for all $\delta \geq \bar{\delta}$. Consequently, from Lemma 4, $q^{e}(s)=q^{*}(s)$ for all $s \in[0,1]$ whenever $\alpha \leq B$ and $\delta \geq \bar{\delta}$. In other words, for a high enough discount rate, if the platform were sufficiently restrained in its pricing, the sellers would deliver the same quality in equilibrium as they would were quality observable. So the failure to achieve even this level of quality when the platform is unrestrained, as shown in Proposition 8, is due to the platform's pricing of access. In particular, the platform captures so much surplus from the sellers that the incentives of the marginal sellers, at least, to maintain a reputation for high quality are eroded and those sellers correspondingly deliver a quality level below the level they would were quality observable. This establishes:

COROLlary 5 But for the profit motive of the platform, there exist discount factors such that all sellers would be active and deliver quality equal to what they would provide were quality observable.

In light of Lemma 5, the platform's problem of setting its profit-maximizing access fee is equivalent to its choosing the $s$ that maximizes

$$
\Psi(s)\left(\underline{q}(s)+B-\frac{c(\underline{q}(s), s)}{\delta}\right) .
$$

Consider the chain

$$
\alpha(s) \equiv \underline{q}(s)+B-\frac{c(\underline{q}(s), s)}{\delta}<B+\underline{q}(s)-c(\underline{q}(s), s)<\pi(s),
$$


where the first inequality follows because $\delta<1$ and the second because, then, $\underline{q}(s) \neq$ $q^{*}(s)$. The following is immediate from (26) and (27):

Proposition 9 Maintain Assumption 2. A platform that charges sellers a fixed access fee earns a lower profit per period in the equilibrium of a repeated-play setting in which seller quality is supported by seller reputation (i.e., the setting of this section) than in equilibrium in a single-play setting in which seller quality is either observable or the platform can set a minimum-quality standard.

\subsection{The Platform Imposes Minimum Quality}

In response to Proposition 9, it is worth asking what happens if the platform can impose and enforce a minimum quality level, $\hat{q}$.

Observe, first, in such a setting the platform can guarantee itself at least a profit of $\Psi\left(s^{*}\right) \pi\left(s^{*}\right)$ : it sets the minimum quality level to $q^{*}\left(s^{*}\right)$ and the access fee to $\pi\left(s^{*}\right)$. This is the same profit that it earns in equilibrium in a single-play setting. This establishes:

Proposition 10 In a repeated-play setting in which the platform can set a minimum level of quality, its equilibrium profit per period cannot be less than its equilibrium profit in a single-play setting. ${ }^{16}$

Given that no seller $s$ will pay a per-period access fee in excess of $\pi(s)$, it follows that the greatest profit the platform can obtain using a fixed access fee is $\max _{s} \Psi(s) \pi(s)$. Moreover, faced with an access fee of $\pi\left(s^{*}\right)$, it is a best response for all $s \leq s^{*}$ to be active. This and the previous proposition establish:

Proposition 11 In a repeated-play setting in which the platform can set a minimum level of quality, its equilibrium profit per period is $\Psi\left(s^{*}\right) \pi\left(s^{*}\right)$. All and only sellers with an index less than $s^{*}$ are active.

The remaining question is what quality do active sellers deliver in this equilibrium? If a seller $s, s<s^{*}$, ruins her reputation, then her per-period utility ever after is

$$
\pi_{0}=B+q^{*}\left(s^{*}\right)-c\left(q^{*}\left(s^{*}\right), s\right)-\pi\left(s^{*}\right)=c\left(q^{*}\left(s^{*}\right), s^{*}\right)-c\left(q^{*}\left(s^{*}\right), s\right) .
$$

It is, thus, credible that she will deliver quality above the minimum if and only if

$$
\frac{1}{1-\delta}\left(B+q-c(q, s)-\pi\left(s^{*}\right)\right) \geq B+q-\pi\left(s^{*}\right)+\frac{\delta}{1-\delta} \pi_{0} ;
$$

equivalently, if and only if

$$
\delta q-c(q, s) \geq \delta\left(\pi^{*}\left(s^{*}\right)+\pi_{0}-B\right)=\delta\left(q^{*}\left(s^{*}\right)-c\left(q^{*}\left(s^{*}\right), s\right)\right) .
$$

\footnotetext{
${ }^{16}$ Note this result and Proposition 11 infra do not require Assumption 2.
} 
Because $q^{*}(s)=\operatorname{argmax}_{q} q-c(q, s), \underline{q}(s)=\operatorname{argmax}_{q} \delta q-c(q, s)$,

$$
\delta(q-c(q, s))>\delta q-c(q, s),
$$

and all functions are continuous, there must exist an $\underline{s} \geq 0$ such that (28) cannot be satisfied for any $q \geq q^{*}\left(s^{*}\right)$ if $s \in\left(\underline{s}, s^{*}\right]$. To summarize:

LEMMA 6 In a repeated-play setting in which the platform sets a minimum level of quality, there is a set of active sellers of positive measure who deliver that minimum level of quality in equilibrium.

The credibility condition in equilibrium when the platform does not set a minimum quality is, from Lemma 5 ,

$$
\delta q-c(q, s) \geq \delta \underline{q}(\widetilde{s})-c(\underline{q}(\widetilde{s}), \widetilde{s}),
$$

where (25) has been substituted into (21). As a point of comparison, suppose that $\widetilde{s}=s^{*}$; that is, the same sellers are active in regime when the platform sets minimum quality and when it does not. Consider the chain and $s<s^{*}$ :

$$
\begin{aligned}
& \delta \underline{q}\left(s^{*}\right)-c\left(\underline{q}\left(s^{*}\right), s^{*}\right)<\delta\left(\underline{q}\left(s^{*}\right)\right.\left.-c\left(\underline{q}\left(s^{*}\right), s^{*}\right)\right) \\
&<\delta\left(q^{*}\left(s^{*}\right)-c\left(q^{*}\left(s^{*}\right), s^{*}\right)\right)<\delta\left(q^{*}\left(s^{*}\right)-c\left(q^{*}\left(s^{*}\right), s\right)\right),
\end{aligned}
$$

where the last inequality follows because $c_{s}>0$. Hence, the rightmost term in (28) is greater than the righthand side of (29). Consider $s \in[0, \underline{s}]$. Utilizing the same logic as in Lemma 4, when the platform sets minimum quality $q^{*}\left(s^{*}\right)$, a seller in that interval chooses the largest quality in $\left[\underline{q}(s), q^{*}(s)\right]$ such that

$$
\delta q-c(q, s)=\delta\left(q^{*}\left(s^{*}\right)-c\left(q^{*}\left(s^{*}\right), s\right)\right) .
$$

That quality cannot be greater than $q^{e}(s)$ as defined by (24) (note the inequality in that expression is the same as (29)) and will be strictly less if $q^{e}(s)<q^{*}(s)$. To summarize:

Proposition 12 Consider two regimes under repeated play. In the first, the platform cannot set minimum quality, but it can in the second. Suppose the same set of sellers are active in equilibrium in the two regimes. Then any seller who delivers quality above the minimum in the second, delivers no greater quality in this second regime than she does in the first; moreover, if, in the first, such a seller delivers quality less than she would were quality observable (i.e., less than $q^{*}$ ), then the quality she delivers in the second is strictly less than in the first.

Although nothing, in general, guarantees that the same set of sellers are active in the two regimes, examples can be constructed in which that holds (in particular, examples in which the platform wants all sellers to be active so $\widetilde{s}=s^{*}=1$ ). Hence, a valid rephrasing of the last proposition is 
COROLlary 6 Settings exist such that, with repeated play, allowing the platform to set a minimum quality will cause some sellers to lower the quality they deliver in equilibrium.

On the other hand, setting a minimum quality can mean some sellers provide higher quality than were there no minimum. Again, suppose $\widetilde{s}=s^{*}$. From Lemma 4, the marginal seller offers quality $q\left(s^{*}\right)$ in equilibrium without a quality minimum. With a quality minimum, she provides quality $q^{*}\left(s^{*}\right)$, which is greater. By continuity, there is a positive measure of sellers who provide higher quality in equilibrium when the platform sets a minimum quality level than when it does not. To summarize:

Proposition 13 Consider two regimes under repeated play. In the first, the platform cannot set minimum quality, but it can in the second. Suppose the same set of sellers are active in equilibrium in the two regimes. Then there exists a set of sellers of positive measure who, in equilibrium, provide higher quality in the second regime than in the first.

\subsection{Agency in a Repeated Setting}

Turning to agency, suppose, first, that the platform does not set a minimum quality standard. Given Assumption 2, a seller will operate even if buyers expect her to deliver zero quality: her payoff of $(1-\tau) B$ exceeds zero. Employing now familiar logic, a seller's promise to deliver quality $q$ is credible if and only if

$$
\frac{1}{1-\delta}((1-\tau)(q+B)-c(q, s)) \geq(1-\tau)(q+B)+\frac{\delta}{1-\delta}(1-\tau) B
$$

Simple algebra reveals (30) is equivalent to

$$
\delta q-\frac{c(q, s)}{1-\tau} \geq 0
$$

Define $q(s, \tau)$ to be the value of $q$ that maximizes the lefthand side of (31). Earlier made assumptions, in particular that $c_{q}(0, s)=0$, ensure that $q(s, \tau)$ is uniquely defined and positive. Because an increase in the "tax" rate is, by (31), equivalent to raising a seller's marginal cost of quality, it follows that $\underline{q}(s, \cdot)$ is a decreasing function for all $s$.

It is readily shown that the quality level

$$
q^{e}(s, \tau) \equiv \max \left\{q \in\left[\underline{q}(s, \tau), q^{*}(s, \tau)\right] \mid \delta q-\frac{c(q, s)}{1-\tau} \geq 0\right\}
$$

exists and is uniquely defined. If $q^{e}(s, \tau)=q^{*}(s, \tau)$, then $q^{e}(s, \cdot)$ is decreasing by Proposition 1. If $q^{e}(s, \tau) \in\left[\underline{q}(s, \tau), q^{*}(s, \tau)\right)$, then

$$
\delta q^{e}(s, \tau)-\frac{c\left(q^{e}(s, \tau), s\right)}{1-\tau}=0
$$

Total differentiation of $(32)$ reveals that $q^{e}(s, \cdot)$ is a decreasing function. To conclude: 
LEMMA 7 In a repeated-play setting with agency, but no minimum quality standard, equilibrium quality is falling with the commission rate (i.e., with $\tau$ ).

Summarizing the analysis to this point:

PROPOSITION 14 In a repeated-play setting with agency, but no minimum quality standard, there is an equilibrium in which all sellers are active and deliver positive quality. Equilibrium quality falls with the platform's commission (tax) rate.

Because $c_{s}>0, s>s^{\prime}$ implies

$$
(1-\tau) q^{*}(s, \tau)-c\left(q^{*}(s, \tau), s\right)<(1-\tau) q^{*}\left(s^{\prime}, \tau\right)-c\left(q^{*}\left(s^{\prime}, \tau\right), s^{\prime}\right)
$$

equivalently,

$$
q^{*}(s, \tau)-\frac{c\left(q^{*}(s, \tau), s\right)}{1-\tau}<q^{*}\left(s^{\prime}, \tau\right)-\frac{c\left(q^{*}\left(s^{\prime}, \tau\right), s^{\prime}\right)}{1-\tau}
$$

Because

$$
(1-\tau) q^{*}(1, \tau)-c\left(q^{*}(1, \tau), 1\right)>0 \Longleftrightarrow q^{*}(1, \tau)-\frac{c\left(q^{*}(1, \tau), 1\right)}{1-\tau}>0,
$$

there exists a $\bar{\delta}<1$ such that for all $\delta \geq \bar{\delta}$

$$
\delta q^{*}(1, \tau)-\frac{c\left(q^{*}(1, \tau), 1\right)}{1-\tau} \geq 0
$$

Using (33), this implies

$$
\delta q^{*}(s, \tau)-\frac{c\left(q^{*}(s, \tau), 1\right)}{1-\tau} \geq 0
$$

for all $s \in[0,1]$. This analysis establishes:

Proposition 15 In a repeated-play setting with agency, but no minimum quality standard, if the parties are sufficiently patient, then there is an equilibrium of the repeated game with unobservable quality that matches the equilibrium of the static game with observable quality in the sense that the platform achieves the same profit in both and each seller offers the same quality in both.

Assuming no capacity to fix a minimum quality, the platform, when limited to agency, cannot do better in the repeated game with unobservable quality than it can with observable quality. On the other hand, as Proposition 15 shows, it need not do worse. This stands in contrast to Proposition 9, which showed that a platform unable to fix a minimum quality and limited to flat-fee access must do worse in a repeated setting with unobservable quality than it would were quality observable.

What if the platform can set a minimum quality level? Given Propositions 5 and 10, the platform must do at least well in a repeated setting as it would in the static game 
with unobservable quality. It may, though, be able to do better. Proposition 5 entails the platform setting a commission rate

$$
\tau^{*}=\frac{\pi\left(s^{*}\right)}{\beta\left(n^{*}\left(q^{*}\left(s^{*}\right)\right), s^{*}\right) n^{*}\left(q^{*}\left(s^{*}\right)\right)} .
$$

Fix that commission rate. If $q^{e}\left(s, \tau^{*}\right) \leq q^{*}\left(s^{*}\right), s<s^{*}$, then seller $s$ chooses the same quality in the repeated game as in the static game and, thus, generates the same revenue for the platform as in the static game. But if $q^{e}\left(s, \tau^{*}\right)>q^{*}\left(s^{*}\right)$, then seller $s$ is choosing a greater quality in the repeated game than she would in the static game and, thus, she generates greater revenue for the platform than she would in the static game. Given seller $s$ 's choice of quality is $\max \left\{q^{e}\left(s, \tau^{*}\right), q^{*}\left(s^{*}\right)\right\}$, it follows that

Proposition 16 Assume that quality is unobservable, but the platform can set a minimum quality. Then the platform does at least as well in a repeated setting as it would in a static setting and it could do strictly better.

This last result stands in contrast to Proposition 11, which showed that, with a flat fee, the platform could do no better in a repeated setting than it could in a static setting.

The difference between Propositions 11 and 16 arises because, with a flat fee, the platform cannot realize any gains if sellers choose quality above the minimum. In contrast, with agency, the platform captures a share of the value created by quality above the minimum. Putting all this analysis together yields

Proposition 17 Consider a repeated-play setting in which quality is unobservable, but the platform can set a minimum quality. The platform's profit using agency weakly exceeds its profit using a flat access fee and strictly exceeds it if $q^{e}\left(s, \tau^{*}\right)>q^{*}\left(s^{*}\right)$ for a positive measure of sellers $s$ (where $\tau^{*}$ is given by (34)).

An analysis of the quality mix offered by sellers in equilibrium under agency and how this varies with the platform's ability to set a minimum quality level is similar to that presented at the end of Section 5.2 and, thus, omitted for the sake of brevity.

\subsection{The Platform Charges Buyers an Access Fee}

If the platform earns its revenue from charging buyers, then the analysis of the play of the sellers is identical to that in Section 5.1, except $\alpha=0$. The principal changes this entails are that (i) all sellers will be active and provide positive quality (recall $q(s)>0$ ); and (ii) by Corollary 5 there exists discount factors such that all sellers provide $q^{*}(s)$ in equilibrium. The second point means, inter alia, that a platform that earns its revenues from charging buyers need not suffer if quality is not observable provided play is repeated and sellers are sufficiently patient.

An analysis when the platform sets a minimum quality level is complex for all the reasons raised earlier. Little of a conclusive nature can be derived; for that reason, as well as for the sake of brevity, no attempt is made to pursue that question here. 


\section{Final Remarks}

This paper has considered a platform, such as an app store or a farmers market, as a regulator of quality in a two-sided setting. Despite the complexity of the situation, the paper is able to derive some definitive results, including some arguably counter-intuitive ones: a platform is indifferent, in a static model, between using agency or charging sellers a flat access fee with unobservable quality when it can set a minimum quality level; a platform that relies on agency can increase its profit by imposing a minimum quality level even when quality is observable to buyers; circumstances exist such that the platform has an incentive to set a higher minimum quality level when it charges sellers than when it charges buyers; repeated play with unobservable quality will result in lower quality than would result with observable quality, regardless of discount factor, if the platform charges sellers a flat access fee; and whereas the platform's preference for agency versus a flat access fee is ambiguous with observable quality, the platform will prefer agency with unobservable quality and repeated play. Nonetheless, it would be fair to say that the paper has only scratched the surface. Many questions remain open.

The paper has sought to emphasize a number of points that warrant further attention. Two are especially critical. First, although reference has been made to Spence (1975) quality distortions, the paper does not study the potential gains the platform could realize by seeking to correct them when its revenues derive from charging buyers for access. In the context of observable quality, the consequences of such regulation would be fairly standard were sellers homogenous with respect to their costs of providing quality. When they are heterogeneous, imposing a minimum quality restriction risks kicking some sellers out of the market, which reduces the surplus buyers can realize and, thus, what the platform can charge them. Studying such tradeoffs is a task for future work.

A second critical point is that the tariffs and pricing mechanisms the platform can employ have been heavily restricted. As noted earlier, an interesting set of issues arise when the platform can charge both sides (e.g., as with iPhone apps, in which Apple charges buyers via the price of the phone and sellers under an agency arrangement). Although such pricing has been explored in a variety of two-sided market contexts (see, e.g., Hermalin and Katz, 2004), issues of quality have been absent. Given the complexity of such pricing models, integrating quality issues is somewhat daunting, but necessary to understand fully the objectives of the platform as regulator of quality.

Another limitation of the existing paper is each seller is a monopoly with respect to her market segment. In reality, many segments have competing sellers (e.g., multiple sellers of aubergines at a farmers market). A consequent issue is, as is well known, that competition can undermine sellers incentives to develop reputations for high quality, which could increase the platform's motive to regulate quality. Another issue is whether, to avoid head-to-head price competition, sellers would wish to vertically differentiate $\grave{a}$ la Mussa and Rosen (1978) - how does such competition affect the setting of minimum quality standards? ${ }^{17}$ On the one hand, the platform, especially if it charges buyers for

\footnotetext{
${ }^{17} \mathrm{~A}$ related question is what would happen if a monopoly seller sought to price discriminate via quality along the lines of Mussa and Rosen and other models of second-degree price
} 
access, has an incentive to encourage competition (thereby raising consumer surplus, which it can capture via the access fee) $;{ }^{18}$ on the other hand, if competition reduces quality, then this can be detrimental to the platform. Another wrinkle with competition is that, depending on the details of that competition, it can raise or lower aggregate profits in the segment. To the extent the platform earns its revenue by charging sellers, this will also have an effect on its interest in encouraging or discouraging competition.

As this discussion - and various comments and footnotes previously made -indicate there remain a number of avenues of future research. Nonetheless, it is to be hoped this paper offers some initial insights as well as road map going forward.

\section{Appendix}

Proof of Lemma 1: Holding $q$ fixed, the $n$ that maximizes (6) is independent of $\tau$ or $s$. Call it $n^{*}(q)$. Note $n^{*}(q)$ maximizes $\beta(n, q) n$. Suppose (3) holds. The cross-partial derivative of $\beta(n, q) n$ with respect to $n$ and $q$ is $n \beta_{n q}(n, q)+\beta_{q}(n, q)>0$; hence, sales are non-decreasing in quality and increasing if the solution is interior. If (4) holds, then maximizing $\beta(n, q) n$ is equivalent to maximizing $\log (\theta q+\kappa)+\log (n b(n))$, the solution to which is independent of quality; so, $n^{* \prime}(q) \equiv 0$.

Proof of Proposition 1: Expression (6) can be rëexpressed as

$$
(1-\tau) \beta\left(n^{*}(q), q\right) n^{*}(q)-c(q, s) .
$$

The cross-partial derivative of (A1) with respect to $\tau$ and $q$ is $-\beta_{q}\left(n^{*}(q), q\right) n^{*}(q)<0$ (using the envelope theorem). So, by the usual comparative statics, $q^{*}$ decreases in $\tau$ ( $q^{*}$ is an interior solution because $c_{q}(0, s) \equiv 0$ and the upper limit on $q$ never binds). The cross-partial derivative of (A1) with respect to $s$ and $q$ is $-c_{q s}(q, s)<0$. So, by the usual comparative statics, $q^{*}$ decreases in $s$. Using Lemma 1 , the rest follows because

$$
\frac{d n^{*}}{d \tau}=n^{* \prime}\left(q^{*}\right) \frac{d q^{*}}{d \tau} \text { and } \frac{d n^{*}}{d s}=n^{* \prime}\left(q^{*}\right) \frac{d q^{*}}{d s} .
$$

Proof of Corollary 2: As a preliminary result, observe, via integration by parts, that

$$
\frac{1}{\Psi(s)} \int_{0}^{s} q^{*}(z, 0) \psi(z) d z=q^{*}(s, 0)-\int_{0}^{s} \frac{\partial q^{*}(z, 0)}{\partial s} \frac{\Psi(z)}{\Psi(s)} d z
$$

Hence,

$$
\frac{d}{d s} \frac{1}{\Psi(s)} \int_{0}^{s} q^{*}(z, 0) \psi(z) d z=\int_{0}^{s} \frac{\partial q^{*}(z, 0)}{\partial s} \frac{\Psi(z)}{\Psi(s)^{2}} \psi(s) d z<0,
$$

discrimination (see, e.g., Hermalin, 2014, for a survey)?

${ }^{18}$ See Economides and Hermalin (2015) for an analysis that, inter alia, explores the incentives of a platform to induce competition. 
where the inequality follows because $q^{*}(\cdot, 0)$ is a decreasing function by Proposition 1 . Necessarily, $s^{*} \leq 1$. Observe

$$
\frac{1}{\Psi\left(s^{*}\right)} \int_{0}^{s^{*}} q^{*}(s, 0) \psi(s) d s \geq \int_{0}^{1} q^{*}(s, 0) \psi(s) d s>\int_{0}^{1} q^{*}(s, \tau) \psi(s) d s,
$$

where the first inequality follows from the preliminary result (recall $\Psi(1)=1$ ) and the second because $q^{*}(s, \cdot)$ is decreasing by Proposition 1 . The first expression in (A2) is average quality in the flat-rate regime and the last is average quality under agency.

Proof of Lemma 4: By rewriting (21), it is clear a seller's promise to deliver quality $q>0$ is credible only if

$$
\delta q-c(q, s) \geq \delta(\alpha-B) .
$$

If that condition fails for $q=q(s)$, it must fail for all $q$ by the definition of a maximum. Hence, no positive level of quality is credible if (23) holds. Because the lefthand side of (23) is positive (recall expression (22)), expression (23) implies $\alpha>B$, which means a seller would prefer to shutdown rather than supply zero quality.

If $(23)$ is reversed, then observe the following chain is valid:

$$
\delta \times(B+\underline{q}(s)-c(\underline{q}(s), s)-\alpha)>\delta \times(B+\underline{q}(s)-\alpha)-c(\underline{q}(s), s) \geq 0
$$

(the second inequality is true because (A3) now holds). Hence, given that $q \mapsto q-c(q, s)$ is a strictly concave function that obtains a maximum at $q^{*}(s)$, it follows that

$$
B+q-c(q, s)-\alpha>0 .
$$

for all $q \in\left[\underline{q}(s), q^{*}(s)\right]$. A seller would wish to be active delivering any $q$ in that interval if her promise is credible; moreover, she would wish to deliver the largest element in that interval. Because (23) is reversed, there exist $q$ in that interval for which the seller's promise is credible; that is, the set in (24) is non-empty. Given the relevant functions are continuous and the inequality that defines the set is weak, the set must have a maximum element; that is, $q^{e}(s)$, as defined by $(24)$, exists.

Proof of Lemma 5: Given (22), the following is true of any $s \in[0,1]$ :

$$
\delta \underline{q}(s)-c(\underline{q}(s), s)>0=\delta(B-B) .
$$

Hence, if

$$
\alpha=\underline{q}(s)+B-\frac{c(\underline{q}(s), s)}{\delta},
$$

then $\alpha>B$. Lemma 4 and the fact that the righthand side of (A4) is decreasing in $s$ entail that if $\alpha$ is given by (A4), then a seller is active if and only if her index does not exceed $s$. It thus follows that the platform would never price access at less than

$$
\underline{q}(1)+B-\frac{c(\underline{q}(1), 1)}{\delta},
$$


because at that price (or less) all sellers would be active and the platform would never price below the maximum price at which all sellers would be active. If the platform priced access at

$$
\underline{q}(0)+B-\frac{c(\underline{q}(0), 0)}{\delta}
$$

or greater, then at most a set of sellers of measure zero would be active, yielding the platform zero profit. Because the platform could make a positive profit setting access according to (A5), it would never set access at or above the level in (A6). Because the righthand side of (A4) is a continuous function and the platform will price between the bounds set by (A5) and (A6), the equilibrium access fee must satisfy (25) for some $\widetilde{s} \in(0,1]$. From Lemma 4 , it is a best response for a seller with index $s>\widetilde{s}$ to be inactive and for a seller with index $s \leq \widetilde{s}$ to be active and deliver quality $q^{e}(s)$.

\section{References}

Akerlof, G. A. (1970), "The Market for 'Lemons': Qualitative Uncertainty and the Market Mechanism," Quarterly Journal of Economics, 84(3), 488-500.

Economides, N., and B. E. Hermalin (2015), "The Strategic Use of Download Limits by a Monopoly Platform," RAND Journal of Economics, 46(2), 297-327.

Hermalin, B. E. (2014), "Uncertainty and Imperfect Information in Markets," in: M. Machina and W. K. Viscusi (eds.), Handbook of the Economics of Risk and Uncertainty, NorthHolland, Amsterdam.

Hermalin, B. E., and M. L. Katz (2004), "Sender or Receiver: Who Should Pay to Exchange an Electronic Message?" RAND Journal of Economics, 35(3), 423-448.

Klein, B., and K. B. Leffler (1981), "The Role of Market Forces in Assuring Contractual Performance," Journal of Political Economy, 89(4), 615-641.

Mussa, M., and S. Rosen (1978), "Monopoly and Product Quality," Journal of Economic Theory, 18(2), 301-317.

Rochet, J.-C., and J. Tirole (2006), "Two-Sided Markets: A Progress Report," RAND Journal of Economics, 37(3), 645-667.

Spence, A. M. (1975), "Monopoly, Quality, and Regulation," Bell Journal of Economics, 6(2), $417-429$.

Sundaram, R. K. (1996), A First Course in Optimization Theory, Cambridge University Press, Cambridge, UK.

Benjamin E. Hermalin

University of California, Berkeley

Department of Economics \#3880

Berkeley, CA 94720-3880 USA

hermalin@berkeley.edu 